\title{
Definir, buscar ejemplos, conjeturar... para probar si un número es feliz
}

\author{
Angelina Alvarado Monroy, Universidad Juárez del Estado de Durango (México) \\ María Teresa González Astudillo, Universidad de Salamanca (España)
}

Recibido el 28 de Octubre de 2013; aceptado el 02 de Febrero de 2014

\section{Definir, buscar ejemplos, conjeturar... para probar si un número es feliz}

\section{Resumen}

Para formar futuros matemáticos es necesario que realicen a tareas en las que deban actuar como tales. En la tarea descrita en este artículo, un grupo de alumnos deben enfrentarse a una definición nueva para ellos: la del concepto de número feliz; deben extraer información que les permita comprenderla para generar ejemplos que respondan con la definición, poder identificar no ejemplos y encontrar organizadores genéricos para construir espacios de ejemplos y finalmente resolver una situación en la que están implicados estos números. Las interacciones dentro del grupo fueron grabadas y analizadas utilizando el modelo RBC-C (Schwarz, Dreyfus \& Hershkowitz, 2009) para documentar cómo tuvo lugar el proceso. Los resultados muestran que, a pesar de algunos errores de los que ellos mismos son conscientes, los alumnos se entusiasmaron con la tarea y consiguieron ir más allá de lo que se les propuso.

Palabras clave: número feliz, construcción de conceptos, definición, generación de ejemplos, generalizar.

Definir, encontrar exemplos, conjecturar... para testar se um número é feliz.

\section{Resumo}

Para treinar futuros matemáticos precisam ser confrontados com tarefas que devem agir como tal. No trabalho descrito neste artigo, um grupo de alunos têm de enfrentar uma nova definição para eles: o conceito de número feliz, devem extrair informação que permita um entendimento para gerar exemplos que atendem à definição, para identificar non-exemplos e encontrar organizadores não genéricos para a construção de espaços de exemplos e, eventualmente, resolver uma situação em que estes números estão envolvidos. As interações en el grupo foram registradas e analisadas utilizando o modelo RBC-C (Schwarz, Dreyfus \& Hershkowitz, 2009) para documentar a forma como o processo ocorreu. Os resultados mostram que, apesar de alguns erros que eles mesmos estão conscientes, os alunos estão entusiasmados com a tarefa e começa a ir além do que está sendo proposto.

Palavras chave: número feliz, construção de conceitos, definição, geração de exemplos, generalização.

Para citar: Alvarado, A., y González-Astudillo, M.T. (2014). Definir, buscar ejemplos, conjeturar... para probar si un número es feliz. Avances de Investigación en Educación Matemática, 5, 5 - 24 
Definir, buscar ejemplos, conjeturar... para probar si un número es feliz

Defining, exemplifying, conjecturing ... to prove if a number is a happy number

\section{Abstract}

To instruct future mathematicians we need to confront them to tasks in which they must perform as if they were really mathematicians. In the task described below a group of students must understand a new definition: the happy number concept definition; they must extract information to generate examples that fit the definition, they must identify non-examples and find generic organizers to construct example spaces and finally solve a situation concerning these numbers. The interactions were first taped and then analyzed using the RBC-C model (Schwarz, Dreyfus, \& Hershkowitz, 2009) to document how the process takes place. The results show that, although they did some conscious mistakes, the students confronted the task enthusiastically and they went on further the initial purpose

Key words: happy number, concept building, concept definition, example generation, generalization.

Définir, trouver des exemples, devinez ... pour tester si un nombre est nombre heureux.

\section{Résumé}

Pour former les futurs mathématiciens ont besoin d'être confrontés à des tâches qui devraient agir en tant que tel. Dans le travail décrit dans cet article, un groupe de étudiants doivent faire face à une nouvelle définition pour eux: le concept de nombre heureux doit extraire les informations qui permettent une meilleure compréhension de générer des exemples qui répondent à la définition, à identifier non-exemples et à trouver des organisateurs génériques pour construire des espaces et finalement résoudre une situation dans laquelle ces nombres sont impliqués. Les interactions ont été enregistrées et analysées en utilisant le modèle RBC-C (Schwarz, Dreyfus \& Hershkovitz, 2009) documente la façon dont le processus s'est produit. Les résultats montrent que, malgré quelques erreurs qu'ils se connaissent, les élèves sont enthousiastes à la tâche et d'apprendre à aller au-delà de ce qui est proposé.

Paroles clés: Nombre heureux, concepts de construction, définition, génération d'exemples, généraliser

\section{Introducción}

Numerosas investigaciones (Moore, 1994; Thompson, 1996; Goetting, 1995; Weber, 2001, entre otros) han puesto de manifiesto las dificultades de los estudiantes universitarios en relación con la noción paramatemática de la demostración (Chevallard, 1985). Moore (1994) considera que algunas de esas dificultades provienen de: la falta de habilidad para formular postulados, la poca comprensión intuitiva de los conceptos involucrados en la demostración, las imágenes inadecuadas que poseen de los conceptos y la ausencia de entrenamiento para generar y usar sus propios ejemplos.

Un primer paso en la producción de demostraciones se da cuando los alumnos comprenden los conceptos involucrados en ellas y utilizan sus definiciones tal como lo hacen los matemáticos ${ }^{1}$. En el nivel universitario se parte del supuesto de que el estudiante debe ser capaz de manejar los conceptos matemáticos y las definiciones implicadas cuando se demuestra. Sin embargo, Selden y Selden (1995) dan cuenta de que los estudiantes no manejan correctamente las definiciones cuando tienen que utilizarlas para organizar argumentos lógicos. De la misma forma, Moore (1994)

\footnotetext{
${ }^{1}$ Es decir como definiciones estipuladas en el sentido de Robinson (1962) lo que significa la explícita y personal conciencia para fundamentar la relación de significado entre alguna palabra y algún objeto, el acto de asignar un objeto a un nombre (o un nombre a un objeto).
} 
menciona que cuando los estudiantes intentan escribir demostraciones formales, no necesariamente están entendiendo su contenido ni muestran evidencias de que conocen cómo usar las definiciones para desarrollar demostraciones.

Edwards y Ward (2004) señalan que, aunque los estudiantes aparentemente parecen entender el papel que tienen las definiciones en matemáticas, en realidad no es así; no conceptúan las definiciones matemáticas como lo hacen los matemáticos y no las usan como lo harían ellos aun cuando puedan enunciarlas y explicarlas correctamente. En este sentido, Bills y Tall (1998) introducen el término definición o teorema formalmente operable para señalar cuándo se usa para crear o reproducir de manera significativa un argumento formal. Consideran que muchos estudiantes no poseen definiciones operables; más bien, cuando tienen que manejar los conceptos, recurren a experiencias anteriores e imágenes de los conceptos que resultan muchas veces inoperables y, cuando tienen cierto éxito, en realidad están usando imágenes que sólo resultan eficientes en ciertos casos.

Un elemento importante tanto en el manejo de las definiciones como en la construcción de demostraciones es el recurso a los ejemplos. Con el objeto de hacer operativa una definición, los alumnos deberían generar ejemplos para mostrar que se cumple alguna propiedad indicada en la definición sin necesidad de probar esa propiedad. Muchas veces, esta forma de proceder se extiende al trabajo con las demostraciones en las que los alumnos se limitan a comprobarla usando ejemplos concretos constituyéndose en simples verificaciones (Alvarado \& González, 2010). Sin embargo, el uso de ejemplos ayuda al estudiante a una posterior generalización y por lo tanto puede ser el medio idóneo para el establecimiento de conjeturas (Watson \& Mason, 2005). Los ejemplos suelen formar parte de lo que se denomina un espacio de ejemplos que "tienen una estructura interna, idiosincrática... y es por esta estructura que los ejemplos se producen” (p. 51) y permite que se puedan establecer relaciones entre ellos en el sentido anterior.

Los estudiantes no utilizan las definiciones de los conceptos matemáticos para demostrar aquellos enunciados en los que se encuentran implicadas. Utilizan más bien un representante concreto del objeto, un ejemplo, alguna fórmula que los represente, $o$ bien sólo consideran alguna característica del objeto matemático (Alvarado \& González, 2010). Aunque son capaces de repetir las definiciones de los conceptos que aparecen en los enunciados de las proposiciones, no son capaces de extraer información relevante de ellas para producir deducciones y transitar entre ellas para demostrar. Además utilizan el lenguaje matemático de forma imprecisa (Alvarado \& González, 2013a).

El objetivo de este estudio es describir el proceso que siguen los alumnos cuando han de manejar la definición de un concepto matemático para construir conocimiento a partir de ella. Para ello analizamos las producciones de un grupo de alumnos usando el modelo RBC-C (Hershkowitz, Schwarz, \& Dreyfus, 2001). En este artículo se describe la actividad situándola dentro de una investigación más amplia y en el contexto en el que se realizó, se analiza el proceso de solución realizado en pequeño grupo y finalmente se establecen algunas conclusiones.

\section{Marco teórico}

Para analizar la construcción de los conceptos matemáticos Schwarz, Dreyfus y Hershkowitz (2009) presentan el modelo teórico Abstracción en Contexto (AiC) 
considerando que se trata de "una actividad vertical de reorganización de constructos matemáticos previos dentro de la matemática y con significados matemáticos para conducir hacía un constructo que es nuevo para el aprendiz" (p. 24).

Este proceso de construcción de un nuevo conocimiento requiere la reorganización de constructos previos interrelacionándolos para dotar de sentido a los nuevos conceptos. Se organiza a través de tres tipos de acciones epistémicas observables: R-acciones (Recognizing), B-acciones (Building-with) y $C$-acciones (Constructing) (RBC). Las $R$-acciones tienen lugar cuando se reconoce un constructo específico previo que los estudiantes poseen y que es relevante para el problema o la situación con la que están tratando. Las $B$-acciones son acciones sobre o con los constructos reconocidos y la combinación de ellos para lograr la comprensión de una situación. Suelen ser acciones de cálculo, representaciones esquemáticas del problema o tarea, justificaciones o razonamientos. Las $C$-acciones se producen cuando se integran constructos previos para producir un nuevo constructo por matematización vertical. Se refieren a la primera vez que se usa o menciona ese constructo nuevo. Mientras que las $R$-acciones y las $B$-acciones se refieren a constructos previos que los estudiantes poseen, las $C$-acciones son evidencias de nuevos constructos.

En este proceso, las $C$-acciones dependen de las $B$-acciones y de las $R$-acciones. Las $C$-acciones obtienen su valor, del establecimiento de relaciones entre las $B$ acciones y las $R$-acciones puesto que suponen el nexo de unión para formar un único constructo. Las $R$-acciones están anidadas dentro de las $B$-acciones, y estas a su vez en las $C$-acciones que pueden anidarse en $C$-acciones de mayor nivel si la primera es un constructo necesario para la segunda (Schwartz, Dreyfus, \& Hershkowitz, 2009).

Cuando el constructo construido se menciona o utiliza después de observada una C-acción se produce la Consolidación que es un proceso a largo plazo y suele emerger en nuevas actividades en las que tiene sentido el uso de ese constructo, por lo que forma parte de un nuevo proceso de construcción de conocimiento. Esta última etapa se caracteriza por evidencia personal, confianza, inmediatez, flexibilidad y cuidado cuando se trabaja con el constructo (Dreyfus \& Tsamir, 2004) y también cuando el lenguaje se muestra cada vez más preciso (Hershkowitz, Schwarz, \& Dreyfus, 2001), aunque para Kidron (2008) y para Gilboa, Dreyfus y Kidron (2011) el aumento en la precisión del lenguaje es característica de la etapa de construcción por sí misma y no únicamente de la etapa de consolidación.

En $\mathrm{AiC}$ a las acciones epistémicas mencionadas se las conoce como modelo RBC (Recognizing, Building with, Constructing) y modelo RBC-C con la segunda C correspondiente a la etapa de consolidación. Este marco teórico constituye al mismo tiempo una herramienta metodológica para el análisis de la dinámica correspondiente a la construcción de conceptos.

En nuestro caso, para poder identificar las acciones epistémicas utilizadas por los alumnos, se desarrolló la actividad en el aula de forma colaborativa en pequeño grupo dando oportunidad a los estudiantes de construir conocimiento compartido y de expresar sus ideas oralmente. Planas y Morera (2011) consideran la interacción como un mediador positivo en la activación y el progreso en los procesos de aprendizaje matemático. 
Las interacciones resultan efectivas si se caracterizan por un intercambio o comunicación real y verdadera, es decir, si los participantes: 1) se comprometen de manera voluntaria en las interacciones sociales con sus compañeros y con su profesor; 2) participan activamente en las interacciones y se involucran con la tarea; 3) aportan ideas y puntos de vista, al mismo tiempo que respetan y valoran las participaciones de sus compañeros y su profesor (taken-as-shared); y 4) no representan una autoridad matemática durante el desarrollo de la interacción (como pudiera ser el profesor). Los alumnos de este estudio realizaron diversas actividades colaborativamente, lo que les permitió ir progresivamente asimilando estas cuatro características y, como se podrá ver más adelante en el análisis, a lo largo de la realización de la tarea a la que se refiere este artículo se puede considerar que sus interacciones fueron efectivas.

Cobb (1995) y Steffe y Wiegel (1992) consideran los puntos 3) y 4) necesarios en interacciones en pequeños grupos, para una buena comunicación y un aprendizaje colaborativo genuino en matemáticas. En este sentido, la relación entre interacción y aprendizaje proporciona de manera simultánea avances por las ideas que se proponen en común en el grupo y limitaciones puesto que a veces los alumnos no son capaces de llegar a resolver la tarea o su resultado es incorrecto.

El marco teórico descrito permite formular la pregunta de investigación como: ¿cuáles son las acciones epistémicas que surgen en el curso de una interacción en pequeño grupo durante el proceso de construcción del concepto de número feliz a partir de su definición?

\section{Metodología}

La actividad descrita a continuación es parte de una investigación más amplia sobre la introducción de la demostración matemática a alumnos universitarios del primer semestre de la Licenciatura en Matemáticas Aplicadas de la Universidad Juárez del Estado de Durango, México. Se diseñaron de hojas de trabajo que guiaran a los alumnos para que organizaran y plasmaran sus ideas, de tal manera, que esta comunicación fuera lo más provechosa posible y se consideraran responsables de su aprendizaje.

El diseño de cada una de ellas se realizó teniendo en cuenta diversos aspectos relativos a la demostración, el objetivo didáctico que se perseguía y algunas otras características como: partir de una situación problemática, formular preguntas que ayudaran a reflexionar sobre el problema o contenido, explorar, manipular, discutir para resolver la situación, plantear preguntas sobre los resultados en forma de retos, extraer conclusiones de la tarea, realizar trabajo extra para los estudiantes que terminaran antes.

Como puede observarse (Figuras 1 y 2), el inicio de la hoja de trabajo está bastante dirigido y hacia el final se vuelve más abierta para que los alumnos tengan la posibilidad de explorar sus ideas.

Concretamente, la tarea presentada en este artículo forma parte de dos hojas de trabajo. La primera se realizó en la primera sesión dedicada a la generación de definiciones (Alvarado \& González, 2013a) como conocimiento previo al de demostración. Posteriormente, se trabajaron diferentes aspectos de la demostración como la identificación de una proposición matemática (Alvarado \& González, 2013b), 
el uso de conectivos lógicos, la generación de ejemplos y contraejemplos, el establecimiento de conjeturas, los métodos de demostración directo, por reducción al absurdo y por contraposición. En la sesión quinta se retoma esta tarea como parte de una hoja de trabajo en la que se trata de la generación de ejemplos y contraejemplos.

El enunciado de la tarea en sus dos partes fue:

Parte I: Definición: Un número es feliz cuando la suma reiterada de los cuadrados de sus dígitos acaba siendo 1.

Proporcionen ejemplos de números felices y justifiquen

Intenten definir número primo feliz

Proporcionen al menos dos ejemplos

Figura 1. Descripción primera parte de la tarea.

Parte II: Después de ver el vídeo mostrado por el profesor o disponible en http://www.youtube.com/watch?v=ee2If8jSxUo intenten contestar al acertijo que ahí aparece ¿qué número sigue en la serie $313,331,367, \ldots$ ? ¿Qué características tienen en común los números de la serie?

¿Qué número sigue? Justifiquen

Después de revisar nuevamente el vídeo, contesten lo siguiente (pueden usar calculadora si así lo desean)

¿Recuerdan la definición de primo feliz? Anótenla

Prueben que 313 es número primo feliz.

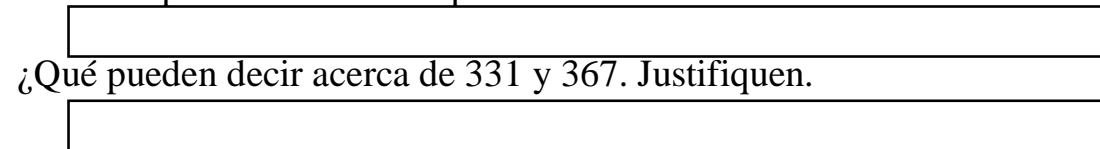

Volviendo a la serie $313,331,367, \ldots$ ¿Qué número sigue? ¿Coincide tu respuesta con la dada antes?

Figura 2. Descripción segunda parte de la tarea.

Para terminar la sesión se les pidió a los alumnos que diseñaran una rutina con algún software de su elección para comprobar si un número es o no feliz.

En esta investigación se realiza un análisis del proceso de construcción del concepto de número feliz en uno de los nueve grupos que se formaron en el aula. Los alumnos de este grupo trabajaron conjuntamente las tareas durante las sesiones habituales de clase. Las discusiones en pequeño grupo fueron video grabadas y transcritas en su totalidad. Para el análisis de dichas intervenciones se establecieron unidades de análisis determinadas por la discusión en torno a un constructo relativo a 
la actividad desarrollada por los alumnos identificando el inicio y el fin de cada una de estas unidades de análisis. Releyendo sucesivas veces hacia adelante y atrás los diálogos de las interacciones se asignaron las acciones epistémicas de cada unidad de análisis. Para asegurar y discutir la asignación efectuada se trianguló esta identificación entre varios investigadores.

\section{Resultados}

Los resultados se han organizado en tres apartados que se refieren: al proceso de comprensión de la definición, a la identificación de una sucesión de números felices y finalmente la puesta en práctica de lo que han aprendido los alumnos. Para cada uno de los apartados se incluye de forma detallada la discusión del equipo A señalando las acciones epistémicas que surgieron en el seno de dichas discusiones.

\subsection{Comprendiendo la definición}

Aunque a los alumnos, además de dar ejemplos de números felices, se les pidió definir número primo feliz, dado que su estrategia fue fusionar las definiciones de número primo y número feliz se ha decidido no incluir la discusión de primo feliz.

En la primera parte de la tarea, a partir de la definición de número feliz, los alumnos debían proporcionar ejemplos de números felices, justificándolos. Inicialmente, el equipo $\mathbf{A}$, mediante una $R$-acción [2] reconoce que si un número es feliz, el que resulta de cambiar de posición sus dígitos también lo es. Sin embargo, esto no les parece adecuado del todo [3] por ser un ejemplo cómodo o bien por no estar convencidos que esto se cumpla siempre. Aun así, se quedan con la idea y esta propiedad la volverán a utilizar en otros momentos [7-9].

[1] Sería este que es primo [eligen el 13] que son todos los primos, son todos los números.

[2] Esto es igual a 1, estoy buscando uno, $; 31$ !

[3] Eso es trampa porque es al revés [de 13].

Al darse cuenta que los números 10 y 13 son felices buscan números cuadrados perfectos que sumen 10 o 13, como 9 y 4, o 1 y 9. El razonamiento (B-acción) que realizan es el siguiente: si me da la suma 10 (como es el caso de 4+6) entonces la siguiente suma será igual a 1, lo cual implica que la raíz de 4 y de 6 deben ser exactas. Como 13 produce la suma $1+9$ buscan combinaciones relacionadas con el 13 y similares con lo que encuentran los números 32,23 y 13, 31. Esto constituye una $C$ acción puesto que esos números no se obtuvieron escogiéndolos al azar y probando si cumple la condición de ser feliz, más bien, los alumnos imponen condiciones ya observadas sobre los dígitos de esos números para conseguir números felices.

[4] Puedo formar con 4 y 6.

[5] No puedo formarlo porque la raíz de 4 es 2, pero no da la de 6. ¿Busco otro número más grande?

[6] ¡Ah ya sé! da nueve y cuatro.

[7] Sería este [exhibe el 23 que es número primo feliz].

[8] Ya sé con el 9 y el 4 Son 13. 
[9] Ajá también 32 [otro estudiante duda] ¿32?

A continuación se produce una $R$-acción [10] al reconocer que $\{11,111,1111$, $11111, \ldots\}$ es un espacio de no ejemplos probando con algunos de ellos. Como con los números con los que prueban no son felices y entre ellos se generan bucles similares deducen que eso es una característica de los números formados por unos. No se dan cuenta de que, por ejemplo, el número con diez 1's sí sería número feliz.

[10] 1111, 111,

Mediante una $R$-acción [18] se dan cuenta que cuando un número es feliz también los números intermedios que se obtienen en el proceso de iteración son felices. Esta propiedad la combinan (R-acción) con la propiedad de los números felices que se obtienen al cambiar de posición sus dígitos [20] y así, en una C-acción, forman espacios de ejemplos que "llevan el 19" o 91, \{19, 91, 82, 68, 133, 139,... . Sin embargo sólo tienen en cuenta los que son primos y descartan de manera implícita 82 y 68 y de manera explícita [22] el 133 por ser divisible entre 19 y entre 7.

[11] El 133.

[12] ¿Lo vas a buscar con todos los números primos? $\neg$

[13] Sería 9 y 9 serían 18 y 1 . A ver, pruébale con el 19 [ignora la pregunta anterior y sigue intentando].

[14] 81 y 1 son 82. A ver. Serían 64 más 4, 68. Se está haciendo más chiquito.

[15] 6 por 6, 36 más 64 serían 100 (Risas).

[16] ¿Cuál era? (Risas). [Responden] El 133.

[17] No, el 19 [otro estudiante duda] ¿19?

[18] Sí, porque el 133 también lleva el 19.

[19] El 139. Uno al cuadrado más 9 más el cuadrado de 9 es uno, es igual a 91. (Risas)

[20] No, pero acuérdate que el 19 también es primo feliz y ya está. (Risas)

[21] Y luego 131. O ¿qué dijiste? ¿133?

[22] 133. Divídelo entre 3. Entre 2, no; entre 3, no; entre 4, no; entre 5, no; 6, 6 hay algo entre 6. A ver entre 7. Sí, es de 7. [Están averiguando si es primo y lo descartan porque es divisible entre 7]

En la siguiente discusión sucede que para un estudiante no queda claro que cuando la suma da un número de un solo dígito distinto de 0 y de 1 entonces el número inicial ya no es feliz. Los argumentos de los compañeros para persuadirle [23, 26 y 28] no resultan convincentes, como se aprecia en [29] donde un alumnos pide una explicación a los compañeros, pero finalmente se convence al mencionar que [30] obtienen un dígito distinto de 1 . De ahí infieren que el único número de un solo dígito que es feliz es el 1 y no exploran los casos del 4 al 9, en los cuales se puede seguir iterando la suma de los cuadrados de sus dígitos. Esta inferencia temprana [R-acción] les lleva a descartar el 7 que sí es un número feliz.

[23] El once. Pero me va a dar dos [se muestra dudoso] ¿Dos?

[24] No. Pero cuando te dé un número, pues ya al cuadrado, ya es responsable ya. 
[25] ¿Cómo?

[26] No, pero cuando tengas otro número, te va a dar 2 y ya.

[27] Es que este tiene dos, dos y al...

[28] Cuando tengas 2 dígitos y ya que te sale uno, el que sea, entonces ya no puedes seguir operando.

[29] ¿Por qué?

[30] Porque ya no se suma éste con otro dígito.

Como 31, 19 y 23 son números primos felices, buscan alguna similitud (B-acción) entre ellos para obtener otros números felices. Encuentran que en todos ellos aparece el dígito 3 (aunque dudan del 19 que no tiene un 3) y buscan otros números que lo contengan para comprobarlo. En esta ocasión su uso impreciso del lenguaje matemático genera cierta confusión y mientras algunos alumnos buscan «múltiplos de $3 »$, otros buscan números en los que aparezca el 3 como dígito, incluso uno trabaja con el 9. Además, centrarse en las similitudes de resultados más que en los procesos (Pedemonte \& Buchbinder, 2011) genera confusión y los ejemplos de números felices [40] y primos $[42,43]$ que consiguen, en realidad no lo son.

[31] 31, 19, 23.

[32] Busca todos los números que le llegan al 3; al 19, no sé...

[33] Múltiplo de 3.

[34] No. No debe tener múltiplos porque si no...

[35] ¡32!

[36] El 32, no. El 32 no se puede.

[37] Porque no es primo, pero sí es feliz.

[38] ¡Así está! Al revés mira [le muestra el 23].

[39] ¿Porque no crees que sea cierto? [le demandan evidencia: A ver cómo, ¿cómo?]

[40] Que los números felices, éste, por ejemplo, el 12, al revés 21. Te dé el 21.

[41] No, ¿21 es un número feliz?

[42] Sí, pero aquí no te está pidiendo que sea primo. ¿Verdad? [Asienten]

[43] Otro primo. El 27.

[44] ¡Ah! A ver 4, 49, es 53.

[45] El 31, el 23 son primos y sí. 25 no.

[46] A ver, vamos con el 34... también da 25.

Los alumnos conjeturan (B-acción) que una vez que encuentran un 5 entre los dígitos [56] se produce un ciclo [57] que permite descartar la posibilidad de que sea número feliz. Esta última conjetura la aplican para verificar que los números 17, 25, $35,45,55,65$ no son números felices. El número del que surge la conjetura es curiosamente el 17, como se puede observar en el siguiente diálogo.

[47] A ver, ponle 17.

[48] 7 por 7,49 y $1,50,5$ por $5,25 \ldots$ 
[49] Es que se va así...

[50] Sí [asienten y lo convencen de desistir]

[51] Es igual a 5 al cuadrado 25 y 4, 29.

[52] 29, 2 por $2,4+81,85$. Mira se va repitiendo; 8 por $8,64+25,89 ; 64+81,145$; [da 4 y ya no es feliz]

[53] ¡Otra vez salió 81!

[54] Ah! Es 14. Ponle 14 y luego, 17, ya regresa. [De 145, $\left.1^{2}+4^{2}=17\right]$

[55] El 35 ya dijimos que no.

[56] ¡Ah! Entonces los que tengan un 5 no cuentan:. 45, 55, 65

[57] Bueno. ¡Hasta ahorita! Todos los que tengan 5 son cíclicos

[58] No, es que no. Precisamente se debe al hecho que tenían 5 [como 17 en la primera suma da 50].

[59] 49 más uno 50 ¡Ay no! ¡Ya valió!, tiene 5

En las siguientes tablas se puede ver cómo emerge (B-acción) la conjetura a través de la verificación de la suma reiterada del cuadrado de los dígitos de números elegidos al azar. Sólo están probando si se cumple o no su conjetura.

Tabla 1.Comprobación con algunos números.

\begin{tabular}{|l|l|l|l|l|l|l|l|l|l|}
\hline Número & 17 & 50 & 25 & 29 & 85 & 89 & 145 & 42 & 20 \\
\hline Suma Cuadrado de dígitos & 50 & 25 & 29 & 85 & 89 & 145 & 42 & 20 & 4 \\
\hline Número & 35 & 34 & 25 & 29 & 85 & 89 & 145 & 42 & 20 \\
\hline Suma Cuadrado de dígitos & 34 & 25 & 29 & 85 & 89 & 145 & 42 & 20 & 4 \\
\hline
\end{tabular}

\begin{tabular}{|l|l|l|l|l|l|l|l|l|l|l|l|}
\hline Número & 45 & 41 & 17 & 50 & 25 & 29 & 85 & 89 & 145 & 42 & 20 \\
\hline Suma Cuadrado de dígitos & 41 & 17 & 50 & 25 & 29 & 85 & 89 & 145 & 42 & 20 & 4 \\
\hline
\end{tabular}

\begin{tabular}{|l|l|l|l|l|l|l|l|l|l|}
\hline Número & 55 & 50 & 25 & 29 & 85 & 89 & 145 & 42 & 20 \\
\hline Suma Cuadrado de dígitos & 50 & 25 & 29 & 85 & 89 & 145 & 42 & 20 & 4 \\
\hline
\end{tabular}

\begin{tabular}{|l|l|l|l|l|l|l|l|l|}
\hline Número & 65 & 61 & 37 & 58 & 89 & 145 & 42 & 20 \\
\hline Suma Cuadrado de dígitos & 61 & 37 & 58 & 89 & 145 & 42 & 20 & 4 \\
\hline
\end{tabular}

Al comparar los resultados y fijarse en el patrón que se repite, los alumnos justifican su conjetura ( $C$-acción) aunque no lo hacen explícito. Además han generado un espacio de ejemplos en torno a dicha conjetura. En principio han podido verificarla con números menores de 100 que contuvieran un 5. Pero además incorporan todos aquellos números que aparecen de manera intermedia en esa verificación, por ejemplo: 16, 17, 29, 37, 61, 34, 89, entre otros. Esto sirve como estrategia (B-acción) para que los alumnos descarten un buen espacio de ejemplos con los que verificar si son o no números felices. 


\subsection{Identificación de una sucesión de números felices}

En la segunda parte de esta tarea (que se hizo en otra sesión distinta de la anterior) se les proyectó parte de un video de una película en el que se menciona la sucesión $313,331,367$ y hay que averiguar el siguiente número para abrir una puerta de seguridad. El profesor les sugirió que todos los números de la sucesión eran números felices [62-63], sin recordarles la definición ni indicarles que además debían ser números primos.

En el equipo A, un estudiante observa [60] que los números de la serie son primos ( $R$-acción). También descubren ( $R$-acción) que al intercambiar las dos últimas cifras del primer número obtienen el segundo [61] y sugieren que el número que sigue es el 376. Al combinar esas dos acciones descartan el número 376 (B-acción) como candidato dado que observan que entre los números 13 y 31 existen más números felices [64], pero no primos felices.

[60] Los primeros números de la serie son primos; es que son...

[61] No. O sea sin el 3, el 67. Pero el 13,31, 67, 76, (...) ah el 76 no es primo.

[62] [...] el profesor nada más nos dijo que eran números felices, no nos dijo que eran primos felices.

[63] Pero es que nada más nos dio una pista.

[64] Pero no, porque igual y el 13 y el 31 entre el 13 y el 31 sí hay algún número feliz pero no hay un número feliz primo.

Las $B$-acciones siguientes giran en torno a la consideración de algunos números para comprobar si son números primos y felices. En el caso del número 373, piensan que si 73 es primo entonces 373 también lo es [65] pero se dan cuenta que no es un número feliz utilizando la conjetura que previamente habían considerado de que al aparecer un 5, el proceso se vuelve cíclico y se puede asegurar que no es número feliz [58 y 59]. Esto constituye una evidencia de una acción de Consolidación puesto que se muestran completamente seguros del constructo que han utilizado y ya no es la primera vez que aparece.

[65] A lo mejor, se me hace que es el 373. No, el 73 es primo

[66] A ver. Hay que checar. A ver, 3 por 3, 9; 7 por 7 que $49+9$ y 9, 67; 36+49, 85. No. Es que tiene un 5

[67] Ajá. Es que se vuelve cíclico

Aunque insisten [68] en que los números mantienen sus características de ser primos felices cuando se prescinde de la primera cifra, encuentran (B-acción) la respuesta correcta [71-73].

[68] Es que si mi teoría es cierta entonces, nos fijamos en los últimos 2 dígitos [de la serie $313,331,367] 13,31,67$ son primos y, aparte, hacen que todo número en conjunto sea un número primo feliz.

[69] Ah! O sea que estos sean primos, pero con el 3 [agregando 3 antes de las cifras 13, 31 y 67] es feliz, pero no necesariamente es primo [asienten].

[70] Bueno supongo que de todos modos sería un primo feliz [asienten]. 
[71] [...] el 379, ¿No? 79 es primo también. A ver 3 por 3, 9 más 81, más 49, 139; 1 más 9 más 81,91 . Sí.

[72] No es que ya te equivocaste desde hace rato [...] Deja, la hago. A ver... 9 más 49 más 81,$139 ; \ldots, 91,81$ más 1 son $82 ; 64$ [más 4], queda 68.

[73] Si ahí dan 100 [número feliz].

[74] 100, entonces ese caso es el que sigue ¿no?

Aún así muestran dudas y al final consideran como solución los dos números, el 376 y el 379.

[75] 379, pero es primero el 376 [muestra desacuerdo otro estudiante]. Pero ese no, ese no es primo.

[76] 379 es primo y feliz... Y es el que sigue.

[77] O sea el 376 no. Si es cierta la teoría de Carlos sigue este [379]. Sino vendría siendo este [asienten].

[78] Es el primo feliz que sigue, consecutivo [apoyan la idea sus compañeros].

[79] Y ya el 376 es solamente feliz.

Comparten respuestas en grupo y revisan nuevamente el video, dejando que avance hasta que se plantea que el siguiente número de la serie debe ser primo y feliz (sin mencionar el número). A continuación prueban que los números 313, 331 y 367 son números primos felices e indican que «376 es el siguiente porque en la serie son números felices consecutivos. Aparte, en los 2 primeros números sus 2 últimos dígitos se intercambian $3 \underline{13} \Rightarrow 3 \underline{31 »}$. La segunda respuesta es que $« 379$ es primo y feliz, es el que sigue».

Finalmente vuelven a la serie $313,331,367, \ldots$ y responden que el número que sigue es 379 .

\subsection{Poniendo a prueba sus conocimientos}

Para terminar, el profesor les pide que utilicen la calculadora TI Voyage, Excel o cualquier otro software que les permita realizar un programa para verificar si un número es feliz, es decir, que construyan una función, que les devuelva la suma de los cuadrados de los dígitos. El profesor les pregunta cómo podrían hacerlo y los estudiantes mencionan que pueden utilizar la representación de un número teniendo en cuenta la posición de las cifras: centenas, decenas, unidades. También señalan que se pueden utilizar los cocientes y residuos al dividir un número entre 10 o 100.

En el equipo A deciden utilizar como apoyo el software de Excel. Al principio se perciben acciones simples de reconocimiento de los conocimientos a emplear: intentan dividir el número entre 10 y elevarlo al cuadrado [83-86] y recuerdan detalles sobre el uso del software. La primera B-acción [87] se produce cuando un estudiante hace reflexionar a los miembros de su equipo al señalar que lo que les interesa es aislar los dígitos del número para lo que deben dividir entre 10 y calcular o bien el residuo o el cociente.

[80] Ahí está un número [en la celda A3 escribe el número 221]. 
[81] Posiciónate en otro lugar [barra para ingresar fórmulas]. Antes tenías que poner paréntesis ¿no?

[82] ¿No es punto y coma?

[83] Ah sí, paréntesis entre 10 [(A3/10)] [otro alumno]. Y ahora ya acabamos. Ahora es éste ¿verdad?

[84] Ajá, al cuadrado ¿porque es al cuadrado? Sí era [(A3/10)^2].

[85] Cómo va a ser lo mismo, entre paréntesis y ya, así ¿no?

[86] No, pero va. El igual va afuera del paréntesis [para generar fórmulas en Excel se usa "=”]

[87] ¿El igual va afuera? Igual, paréntesis, pero entero o residuo de A3, punto y coma $\left[=\left(\right.\right.$ Entero $(\mathrm{A} 3 ; 10)^{\wedge} 2$ o bien $\left.=(\operatorname{Residuo}(\mathrm{A} 3 ; 10))^{\wedge} 2\right]$.

Como lo que tratan es de obtener la cifra de las decenas, señalan que si el número que tienen en la celda $\mathrm{A} 3$ es 221 se divide entre 10 y se toma la parte entera, no se tendría un 2 sino un 22; esto constituye otra B-acción [88]. Mediante B-acciones anidadas [89] deciden utilizar el residuo de la división entre 100 y restarle el residuo de la división del número entre 10, llegando así a una C-acción [90] donde ya se construye una fórmula que les permite aislar el dígito de las decenas. Como algún compañero parece que no lo entiende, se vuelve a explicar [94] y proceden a elevar al cuadrado. Aun cuando tienen la expresión general, buscan verificar con algún otro ejemplo particular [96].

[88] No, pero queremos que sea 2.

[89] Por eso... así, sería este con el residuo.

[90] No mira. Es que va a estar más largo y luego, entre paréntesis A3, punto y coma $100 \ldots$, cierra el paréntesis y luego, a eso hay que restarle lo que nos dio aquí y dividirlo entre 10 y así nos va a quedar el 2 [se refiere al 2 de las decenas de 221 y propone la fórmula (Residuo (A3;100))-(Residuo(A3;10))/10)].

[91] Es este. Otro paréntesis.... sería B3.

[92] No es así. No. Es que... ponle, hay un menos [pregunta con duda el estudiante] ¿Un menos?

[93] Ajá, es igual.... A ver, a este le restaste este y todo eso hay que dividirlo entre 10.

[94] A 221 entre 100, me quedaría 2 y sobran... 21, menos 1 te queda 20 y divides entre, entre 10, te queda 2 [verifican en la fórmula dada antes].

[95] Al cuadrado, porque acá le estábamos sacando la raíz a uno... Ah no. Aquí lo estábamos elevando al cuadrado. Aquí le sacamos la raíz ¿no? [residuo lo confunde con raíz].

[96] [...] lo que estamos viendo aquí es restarle. Por ejemplo, aquí tenemos 3, le vamos a restar $9[\ldots]$, si tenemos 3, le vamos a restar 9 [ensaya otro número para entender] Ah, ya te entendí. Sí es cierto.

En el diálogo se muestra una fuerte asociación entre las operaciones "raíz" y "división". De manera insistente los alumnos utilizan el término raíz para referirse al residuo de una división [97-98-100]. 
El resto de la interacción muestra una revisión de sintaxis de instrucciones en Excel para concretar el contenido que habrá de ir en la fórmula obtenida en la $C$ acción de [90].

[97] Entonces, este sería,..., dividir éste sería dividir. Y la raíz [residuo] de A3. Aquí sería menos la raíz [residuo] de A3.

[98] Cómo se pone la raíz, Sqrt... se pondrá aquí, así [le contestan]. Quien sabe, pero es residuo.

[99] A ver... con minúsculas... sí será así. Mejor hay que poner todo así ¿?

[100] No pues. Pero ¿Cuál? Quítale el sqrt y luego métete adentro del paréntesis y luego, aquí.

A continuación tratan de encontrar una expresión para obtener el dígito de las centenas al cuadrado y para ello consideran ( $R$-acción) el residuo de la división entre 100 , y basándose en el trabajo previo piensan dividir el número entre 1000. Ocurren entonces dos B-acciones al pensar en que al dividir entre 1000 el residuo sería el mismo número por ser de 3 cifras $\mathrm{y}$, a eso, tendrían que restarle el residuo de la división del número entre 100, es decir los dos últimos dígitos [102-103]. Estas acciones les conducen a una C-acción [108-109] donde definitivamente deciden dividir entre 100 para obtener el dígito de las centenas en un número de 3 cifras y luego deben elevarlo al cuadrado.

[101] A3 [más de una voz]. Coma, punto y coma... 100.

[102] Pero nos va a quedar lo mismo... 221...entre 1000. Que nos quede [residuo] 221.

[103] Más bien sería eso de A3, menos el residuo de todo esto con 100 ¿no?

[104] Ah! OK. Sería A3... menos, sería el residuo de este... Es que les vas a restar 21 ¿no?

[105] Menos el residuo de A3 entre 100 ¿no?

[106] Sí A3 menos el residuo de éste, de A3 entre 100, que sería 21.

[107] Entre paréntesis. El residuo vendría siendo 21 [afirman].

[108] A3 menos 21 serían 200 y luego así y luego todo eso entre... entre 100 ¿verdad?

[109] Y ya todo eso al cuadrado $\left[(((\operatorname{Residuo~}(\# ; 1000))-(\operatorname{Residuo}(\# ; 100)) / 100))^{2}\right]$

Finalmente, se obtiene de manera directa una $C$-acción [110] que corresponde con la expresión para encontrar el dígito de las unidades al cuadrado, y otra $C$-acción [111] que suma las expresiones parciales para establecer una fórmula que les devuelva la suma de los cuadrados de los dígitos de un número (Figura 3). 


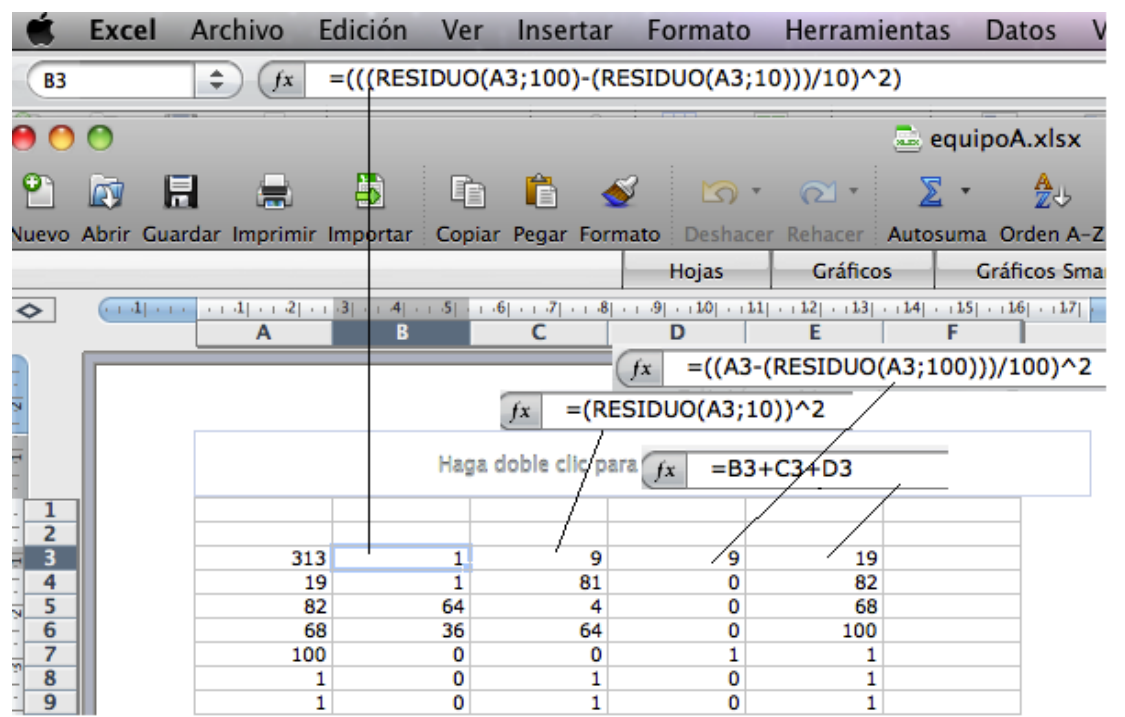

Figura 3. Hoja Excel realizada por el equipo A.

Hasta el momento [112], únicamente se tiene la primera de las sumas del cuadrado de los dígitos. El proceso tiene que reiterarse para poder determinar si el número es feliz. Mediante una R-acción [114] los alumnos reconocen que en Excel es posible hacer que a partir del resultado obtenido se repita el proceso cuantas veces sea necesario, pero recurren al profesor para que les dé alguna indicación. Deciden que 30 iteraciones [116] pueden ser suficientes para verificar que un número sea feliz lo que constituye una $B$-acción que permite la iteración del proceso.

[110] Y en D3, residuo del número con [la división entre] 10, todo esto al cuadrado (...). Sí y ya le vamos entendiendo muchachos.

[111] Y ahí ponle la suma de B3 más C3 más D3.

[112] ¿Qué le ponemos para que salga la función, Carlos? [Hasta el momento sólo tienen la primera suma del cuadrado de los dígitos].

[113] Y luego acá ponle ese. Que sería eso mismo igual A3... A3.

[114] No. A este (...), A3 y luego aquí vendría siendo (...) ¿Profesor cómo lo hacemos desde el mismo las otras sumas? [El maestro les indica que seleccionen las celdas y del extremo inferior izquierdo con click sostenido para que se copien las fórmulas en las celdas hacia abajo y puedan realizar las sumas reiteradas].

[115] Ponte acá, donde aparezca la crucecita [...], en la blanca [...] y con esa destiéndete hasta abajo.

[116] 30, digamos.

Después de cumplir con la tarea en el equipo se percibe una gran satisfacción al haber encontrado su propia fórmula. Comprueban que funciona con diferentes números $(89,313,23,80,29,139, \ldots)$ mostrando entusiasmo cuando logran dar con un número feliz (377). No se incluyen todos los ejemplos que ponen porque resultaría excesivamente reiterativo.

[117] El 89, aquí el 1 al cuadrado, el 8 al cuadrado ¿? Y ya se cicló.... A ver vamos a poner otro... ¿Cuál? 
[118]... El 230. oohhh.

[119] El 23 verdad [si le agregan ceros sigue siendo feliz].

[120] El 130 también.

[121] 213? ...103. ¡Listo! Creo que con todos así.

[122] El cero. ¡Ah [...] verdad! son puros ceros (risas) Qué listotes somos (Risas)

Esta tarea trascendió al aula y al día siguiente los estudiantes mostraron al profesor que la fórmula funcionaba también si lo hacían con la parte entera (cociente) de la división:

Tabla 3. Fórmula para la comprobar si un número es feliz.

(entero(número/100) $)^{2}+(\text { entero((número-(100*entero(número/100)))/10) })^{2}+($ número$(\text { entero(número/10)*10) })^{2}$

Incluso un alumno hizo un programa en lenguaje Pascal para poder establecer si un número es feliz o no.

\section{Conclusión y discusión}

La discusión por equipos consideramos que fue efectiva (Cobb, 1995 y Steffe \& Wiefel, 1992) y permitió que la construcción y exploración de ejemplos se fuera ampliando, encontrando patrones que permitieran la integración de los mismos en ciertas agrupaciones constituyendo lo que se llama un espacio de ejemplos (Watson \& Mason, 2005). Esta tarea constituyó, en cierta manera, un desafío; los alumnos se aventuraron cada vez más en la búsqueda de ejemplos más complejos y estructurados que les permitiera construir algunas conjeturas (B-acciones) para obtener $(C$ acciones) espacios de ejemplos cada vez más generales.

Los alumnos propusieron conjeturas que se fortalecían al probar con números de más cifras. Por ejemplo, si en el proceso iterativo aparece un 5 en uno de los pasos de la suma de los cuadrados de los dígitos se puede asegurar que el número no será feliz. Así lo encontramos en los comentarios de las transcripciones de las grabaciones: «se cicla» 0 «tiene un cinco ya no».

La construcción de ejemplos ayudó a los estudiantes a construir conjeturas y verificarlas, en principio sólo observando resultados, pero poco a poco se van fijando en la estructura o en el proceso en que se fueron obteniendo, con lo que surgen además de ejemplos, contraejemplos y no ejemplos.

Los alumnos evidenciaron dos bloques de $\mathrm{C}$-acciones en la ejecución de esta tarea. Ligadas a la primer parte de comprensión de la definición, su primera C-acción constituyó la generación de números felices a partir de cuadrados perfectos. Otra Cacción fue la de considerar los números intermedios en el proceso de comprobación de que un número era feliz, también como números felices. Y finalmente, la última C-acción de este bloque, está relacionada con la conjetura para descartar números cuando aparece algún 5 en el ciclo de comprobación. Estas C-acciones se puede 
considerar que son independientes unas de otras y han permitido a los alumnos un conocimiento relacional de los números felices.

El segundo bloque de C-acciones está ligado a la generación de un programa informático para comprobar si un número es o no feliz. Podemos identificar en este sentido tres $\mathrm{C}$-acciones para realizar el cálculo del cuadrado de cada uno de los dígitos y una C-acción de orden superior que se apoya en la anterior para determinar la suma de los cuadrados y generar las reiteraciones de ese proceso que permiten averiguar si un número es feliz.

En esta tarea se produjo la Consolidación esperada en la segunda parte correspondiente a la generación de números primos a partir de un vídeo dado que se reutilizaron las $C$-acciones que habían sido adquiridas previamente.

El profesor no esperaba que este tipo de actividades estimularan a los estudiantes a seguir indagando. Ni estudiantes, ni profesores están acostumbrados a este tipo de dinámicas, ya que en la universidad se privilegian prácticas más formales. El énfasis está puesto más en la demostración formal y no se le da importancia al contenido estrechamente ligado con esta tarea como es el desarrollo intuitivo de ideas, la visualización, la organización de información en tablas, las prácticas para que los estudiantes exploren y encuentren patrones que les permitan producir conjeturas o crear sus "propias fórmulas" como en este caso.

Como conclusión se considera que: 1) la generalización es fundamental para el pensamiento matemático; 2) la generalización algebraica es un elemento primario hacia la abstracción matemática y puede ser desarrollada a partir del trabajo con patrones o regularidades que favorecen la articulación de la generalización en situaciones cotidianas y que 3) para aprender el lenguaje algebraico es importante que el alumno tenga algo que comunicar, para lo que se necesita percibir un patrón o una regularidad y después intentar expresarlo y comunicarlo a alguien.

De acuerdo con lo observado en esta sesión se sugiere a los profesores, al igual que Blanton y Kaput (2003), que fomenten en sus alumnos la cultura de modelar, explorar, comentar, predecir, suponer y poner a prueba sus ideas, además de poner en práctica sus habilidades y tener la oportunidad de descubrir patrones, realizar conjeturas o generalizaciones sobre hechos y relaciones matemáticas con su respectiva justificación.

Agradecimientos: Esta investigación ha sido subvencionada en parte con un proyecto del Plan Nacional de Investigación del Ministerio de Investigación y Ciencia de España con referencia EDU2011-29328.

\section{Referencias}

Alvarado, A., \& González, M.T. (2010) La implicación lógica en el proceso de demostración matemática: estudio de un caso. Enseñanza de las Ciencias, 28(1), pp. $73-84$

Alvarado, A., \& González, M.T. (2013a) Interactive reconstruction of a definition. En B. Ubuz, C. Haser, M.A. Mariotti (eds.) Proceedings of the Eighth Congress of the European Society for Research in Mathematics Education, 2276-2285. Antalya, Turquía 
Alvarado, A., \& González, M.T. (2013b) Generación interactiva del conocimiento para iniciarse en el manejo de las implicaciones lógicas. RELIME, 16(1), 37-63.

Bills, L., \& Tall, D.O. (1998) Operable Definitions in Advanced Mathematics: The case of the Least Upper Bound, Proceedings of PME 22, Stellenbosch, South Africa, 2, 104-111.

Blanton, M., \& Kaput, J. (2003) Developing elementary teachers' algebra eyes and ears. Teaching Children Mathematics, 10(2), 70-77.

Cobb (1995) Mathematical learning and small-group interaction: Four case studies. In P. Cobb \& H. Bauersfeld (Eds.) The Emergence of Mathematical Meaning: Interaction in Classroom Cultures (pp. 25-129), Lawrence Erlbaum, Hillsdale.

Chevallard, Y. (1985). La transposition didactique; du savoir savant au savoir enseigné. Paris: La Pensée Sauvage.

Dreyfus, T., \& Tsamir, P. (2004). Ben's consolidation ok knowledge structures about infinite sets. Journal of Mathematical Behavior, 23, 271-300.

Edwards, B., \& Ward, M. (2004). Surprises from Mathematics Education Research: Student (Mis)use of Mathematical Definitions. The Mathematical Association of America Monthly, 111, 411-424.

Gilboa, N. Dreyfus, T. \& Kidron, I. (2011). A construction of a mathematical definition: the case of parabola. Proceeding of the 35PME (2), p.425-432. Turquía.

Goetting, M. (1995). The college students' understanding of mathematical proof (Doctoral dissertation, University of Maryland, 1995). Dissertations Abstracts International, 56,3016A.

Hershkowitz, R., Schwarz, B.B. \& Dreyfus, T. (2001). Abstraction in context: Epistemic actions. Journal for Research in Mathematics Education, 32, 195-222.

Kidron, I. (2008) Abstraction and consolidation of the limit procept by means of instrumented schemes: The complementary role of three different frameworks. Educational Studies in Mathematics, 69, 197-216.

Moore, R. C. (1994) Making the transition to formal proof. Educational Studies in Mathematics, 27(3), 249-266.

Pedemonte, B., \& Buchbinder, O. (2011). Examining the role of examples in proving processes through a cognitive lens: the case of triangular numbers. ZDM, 43(2), 257-267.

Planas, N., \& Morera, L. (2011). La argumentación en la matemática escolar: dos ejemplos para la formación del profesorado. In E. Badillo, L. García, A. Marbà \& M. Briceño (Eds.), El desarrollo de competencias en las clases de ciencias y matemáticas (pp. 275-300). Mérida: Fondo Editorial Mario Briceño Iragorry. Universidad de los Andes.

Robinson, R. (1962). Definitions. London: Oxford University Press.

Schwarz, B.B., Dreyfus, T. \& Hershkowitz, R. (2009). The nested epistemic actions model for abstraction in context. En B.B. Schwarz, T. Dreyfus \& R. Hershkowitz (Eds.), Transformation of Knowledge through Classroom Interaction (pp. 11-42). London, UK: Routledge. 
Selden, J., \& Selden, A. (1995). Unpacking the logic of mathematical statements. Educational Studies in Mathematics, 29, 123-151.

Steffe, L.P., \& Wiegel, H.G. (1992) On reforming practice in mathematics education. Educational Studies in Mathematics, 23(5), 445-465.

Thompson, P. W. (1996). Imagery and the development of mathematical reasoning. In L. P. Steffe, B. Greer, P. Nesher, \& G. Goldin (Eds.), Theories of learning mathematics (pp. 267-283). Hillsdale, NJ: Erlbaum.

Watson, A., \& Mason, J. (2005). Mathematics as a constructive activity: Learners generating examples. Mahwah, NJ: Lawrence Erlbaum Associates.

Weber, K. (2001) Student difficulty in constructing proof: The need for strategic knowledge. Educational Studies in Mathematics, 48(1), 101-119.

\section{Referencias a los autores}

Angelina Alvarado Monroy, Universidad Juárez del Estado de Durango (México). aalvarado@usal.es

María Teresa González Astudillo, Universidad de Salamanca (España). maite@usal.es 


\title{
Defining, exemplifying, conjecturing... to prove if a number is a happy number
}

\author{
Angelina Alvarado Monroy, Juárez University of Durango State (México) \\ María Teresa González Astudillo, University of Salamanca (España)
}

In this paper we present part of a larger research about proof that analyzes how university undergraduate students of mathematics handle new definitions as a previous step to make a whole proof by their own. For future mathematicians to be able to construct proofs, they must understand what a correct mathematical definition is and how to use it properly. This requires students to have a clear idea of what a mathematical definition is. But, as the research literature has shown, the students do not understand the content of relevant definitions properly and how to use them for writing proofs (Moore, 1994).

To instruct future mathematicians we need to confront them to tasks in which they must perform as if they were really mathematicians. In the task describe in this paper a group of students must understand a new definition: the happy number concept definition; they must extract information to generate examples that fit the definition, they must identify non-examples and find generic organizers to construct example spaces and finally they must solve a situation concerning these numbers.

The task was designed by the researchers and was put into practice during two of the ten sessions that corresponds to the whole research: the first one dealt with different concept definitions and the fifth session addressed the use of examples and counterexamples. The interactions between the students were first taped and then analyzed using the RBC-C model (Schwarz, Dreyfus \& Hershkowitz, 2009) to document how the process took place.

The results showed that, although they did some conscious mistakes, the students confronted the task enthusiastically and they went on further the initial purpose. They explored the new definition generating examples to organize their own example space and they made and checked conjectures to solve the task.

Using the methodological frame we might identify the epistemic actions shown during the students interactions linked to this task. This epistemic actions were classified in two groups one of them explains how students understand the new definition and the other describes the construction of a computer program that could generate happy numbers. We have confirmed that the students reached the Consolidation of this concept in the second part of the task.

Finally, we argue that during the interactions to have negotiated definitions in small-groups helped the students to extend their understanding about the meanings of the terms. On the other hand, this activity suggests an issue that should be taught and learned in the classrooms in order to improve the mathematical communication, instead of assuming that students already have acquired those skills. 